Check for updates

Cite this: Mater. Adv., 2020, 1,2501

Received 4th August 2020, Accepted 15th September 2020

DOI: $10.1039 / \mathrm{d} 0 \mathrm{ma00566e}$

rsc.li/materials-advances

\title{
Nanosponges for the protection and release of the natural phenolic antioxidants quercetin, curcumin and phenethyl caffeate
}

\author{
Susanna Guernelli, ${ }^{a}$ Alice Cariola, ${ }^{a}$ Andrea Baschieri, ${ }^{a}$ Riccardo Amorati (D) *a and \\ Paolo Lo Meo (D) *b
}

\begin{abstract}
The inclusion of polyphenols into nanoporous materials may significantly improve their application as radical trapping agents for therapeutic purposes. In the present work, nanosponges based on hypercross-linked cyclodextrins and calixarenes (NS1-NS3) were used as carriers of three natural phenolic antioxidants: quercetin (Que), curcumin (Cur) and phenethyl caffeate (Phec). Good w/w loadings, namely 7.3\% for the Que-NS1 composite, $17.3 \%$ for Cur-NS2 and $12.9 \%$ for Phec-NS3, were achieved. The release kinetics and the inhibition rate constants $\left(k_{\text {inh }}\right)$ of the reaction with alkylperoxyl radicals $\left(\mathrm{ROO}^{\bullet}\right)$ in $0.1 \mathrm{M}$ phosphate buffer solutions at $\mathrm{pH} 7.4$ were studied and indicated better antioxidant activity as compared to the phenols alone. The three phenols suffered a dramatic reduction of $k_{\text {inh }}$ upon increasing their concentration, as a result of their degradation. The inclusion into the nanosponges protected the antioxidants and provided a controlled release in an aqueous medium. Hence, different applications can be envisaged, in food and pharmaceutical technology fields.
\end{abstract}

\section{Introduction}

Antioxidant supplementation is considered an effective method to modulate the redox processes that are associated with aging and various pathological conditions. ${ }^{1}$ Increased oxidative stress and related systemic inflammatory processes are often present in degenerative diseases ${ }^{2}$ and in abnormal immune responses, such as those recently observed in COVID-19-related pneumonia. ${ }^{3}$ Food-grade antioxidants from plant-derived extracts are among the first-choice ingredients for antioxidant therapies because they are believed to be safer than novel synthetic drugs, as they are already present in the human $\operatorname{diet}^{4}$ Natural polyphenols such as curcumin, resveratrol, phenolic acids and flavonoids are among the most popular compounds used as potential modulators of the free radical-induced cellular processes. ${ }^{4,5}$ However, one important problem that restricts the therapeutic potential of polyphenols is their oxidative instability in water solution and low bioavailability. ${ }^{6,7}$ The use of nanodelivery systems has allowed the enhancement of the solubility and stability of bioactive phytochemicals. ${ }^{8-10}$ In particular, the bioavailability can be also 5-10 times higher than that of the native formulation. ${ }^{11}$ Recent studies report several examples of

\footnotetext{
a Dipartimento di Chimica "G. Ciamician”, Università degli Studi di Bologna, Via S. Giacomo 11, 40126 Bologna, Italy. E-mail: riccardo.amorati@unibo.it

${ }^{b}$ Dipartimento STEBICEF, Università degli Studi di Palermo, Viale delle Scienze,

Ed. 17, 90128 Palermo, Italy. E-mail: paolo.lomeo@unipa.it
}

antioxidants in nanoformulations, such as plant-based bioactive compounds (resveratrol, curcumin, quercetin, genistein, etc.) encapsulated in lipidic or polymeric nanosystems, ${ }^{7}$ which have shown efficacy in preclinical studies, especially in relation to the increased bioavailability of the antioxidant. ${ }^{10}$ In general, the development of nanotechnology-based applications for targeted delivery of bioactive phenolic compounds with antioxidant properties to treat chronic diseases is an urgent task of nanomedicine research. ${ }^{12}$

Nanosponges (NSs) are materials with a nanosized porosity ${ }^{13}$ that represent an emerging tool to improve the solubility and stability of sparingly water soluble drugs. ${ }^{14-16}$ Nanosponges are hyper-cross-linked polymers or co-polymers made up of typical supramolecular host species, such as cyclodextrins (CDs) or calixarenes (CAs) as the monomers, covalently held together by means of suitable linker units. Native CD NSs can be obtained by reacting the $\mathrm{CD}$ synthon with double electrophiles ${ }^{17}$ such as bis-isocyanates ${ }^{18}$ whereas per-halo-CD derivatives can be reticulated by reaction with polyamine nucleophiles. ${ }^{18,19}$ More recently, CA NSs have been obtained according to a "click" strategy, by reacting propargyloxy-CAs with bis-azidoalkanes under the well-known CuAAC reaction conditions. ${ }^{20}$ The same approach has been exploited to prepare mixed cyclodextrincalixarene NS materials (CDCANSs, Fig. 1). ${ }^{21,22}$ The latter ones present a disordered structure that is permeable to aqueous solutions and able to reversibly absorb and release diverse organic guest species (including dyes ${ }^{19}$ and antimicrobial agents ${ }^{20}$ ), which 


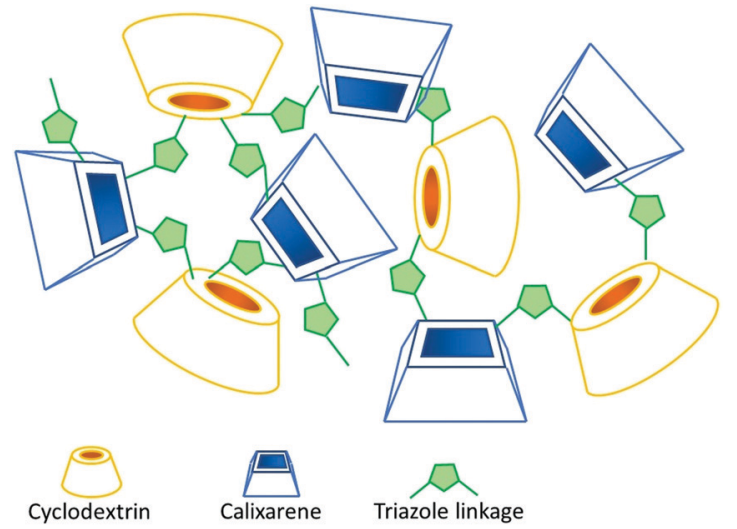

Fig. 1 Exemplificative representation of the disordered structure of CDCANSS.

can be accommodated into both the cavities of the host units and the nanochannels. Of course, the morphological, mechanical and physico-chemical properties of the material critically depend on the chemical nature of both the starting synthon(s) and the linker, as well as on their molar ratio. By a sensible choice of the synthetic strategy, it is possible to obtain materials with tunable properties and, in particular, to achieve sensitivity to external stimuli such as $\mathrm{pH}$ variations. ${ }^{19,21-25}$

CD-based NSs have been proposed to improve the bioavailability of polyphenols, such as resveratrol, ${ }^{26,27}$ oxyresveratrol,,${ }^{27,28}$ curcumin ${ }^{29}$ quercetin, $^{30}$ ferulic acid, ${ }^{31}$ rutin, ${ }^{32}$ phlorizin $^{32}$ and chlorogenic acid. ${ }^{32}$ However, despite the growing interest toward NSs as carriers of polyphenols, the antioxidant activity of these materials has been seldom investigated. ${ }^{27-29}$ To the best of our knowledge, the efficiency of the reaction of polyphenols loaded into nanosponges with alkylperoxyl radicals ( $\left.\mathrm{ROO}^{\bullet}\right)$, which are the chain carrying ones of peroxidation in bio-organic systems, ${ }^{9,33}$ has never been studied in detail. Most popular assays that are used to measure the antioxidant activity of new materials rely on the decay of coloured stable radicals such as 2,2-diphenyl-1-picrylhydrazyl radical $\left(\mathrm{DPPH}^{\circ}\right)$ in alcohol solvents. These methods, although providing an easy estimate useful for screening purposes, have been subjected to criticism ${ }^{34}$ because they do not describe the ability to inhibit the peroxidation of an oxidizable substrate (for instance, of unsaturated lipids ${ }^{9}$ ) and have little relationship with the radicals and solvents involved in biological systems. ${ }^{35}$ So, in the context of our ongoing studies on the peroxyl radical trapping of nanosized/nanoporous materials, ${ }^{36-38}$ we were interested in characterizing the antioxidant effect of polyphenols included in NSs. Herein, the preparation and properties of composites constituted by quercetin (Que), curcumin (Cur) or phenethyl caffeate (Phec, Fig. 2) included into CD or mixed CD-CA nanosponges are presented, along with a detailed investigation of their release kinetics and reactivity with alkylperoxyl radicals in a buffered water solution. The polyphenols considered herein are promising therapeutic agents and are representative of some of the main classes of naturally occurring phenols. ${ }^{39-41}$ In the present work, we chose to test different NSs having diverse chemical nature, in view of the possible tunability of their inclusion and release properties.

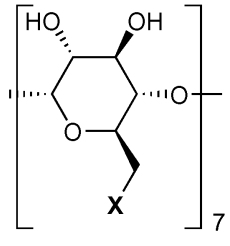

$\beta C D: \mathbf{X}=\mathrm{OH}$ $A_{7} C D: X=N_{3}$<smiles>O=c1c(O)c(-c2ccc(O)c(O)c2)oc2cc(O)cc(O)c12</smiles><smiles>COc1cc(/C=C/C(=O)/C=C(O)/C=C/c2ccc(O)c(OC)c2)ccc1O</smiles>

Cur<smiles>O=C(/C=C/c1ccc(O)c(O)c1)OCCc1ccccc1</smiles>

Phec
Fig. 2 Structures of the polyphenols Que, Cur and Phec

Fig. 3 Structures of the synthons used in the preparation of NS materials.

In particular, we first considered a simple CD-based material obtained by reticulating native $\beta$-CD with hexamethylene-bisisocyanate (hmbi) and then two CD-CA-NS materials obtained by reacting heptakis-(6-azido)-(6-deoxy)- $\beta \mathrm{CD}\left(\mathrm{A}_{7} \mathrm{CD}\right)$ with tetrapropargyloxy-calix[4]arene $\left(\mathrm{P}_{4} \mathrm{C}[4] \mathrm{A}\right)$ in either $2: 1$ or $1: 2$ equivalent/equivalent ratio (Fig. 3; the three materials will be herein after indicated as NS1, NS2 and NS3, respectively). The synthesis and properties of the chosen materials have been already reported. ${ }^{22,42,43}$

\section{Results and discussion}

\section{Loading and release of nanosponges}

By means of absorption tests at two different $\mathrm{pH}$ values, namely 4.4 and 6.7, we preliminarily verified that the selected materials were able to absorb the guest molecules from aqueous buffered solutions (for the sake of clarity, it is worth mentioning here that all the materials used in this work presented a $<150 \mu \mathrm{m}$ granulometry). The two $\mathrm{pH}$ values were chosen for consistency with previous studies and in general have been found to be the most suitable for absorption and release studies. The results obtained (presented in Table 1) show an excellent sequestration efficiency, larger than $80 \%$, in all the cases examined; in particular, a nearly quantitative absorption for curcumin is always observed. In most cases a negligible variation in inclusion efficiency was observed upon changing the $\mathrm{pH}$, with the remarkable exception of Que in materials NS2 and NS3. 
Table 1 Uptake of phenolic antioxidants from a buffered water solution by the nanosponges at different $\mathrm{pH}$ values $^{\mathrm{a}}$

\begin{tabular}{|c|c|c|c|c|c|c|}
\hline \multirow[b]{2}{*}{$\mathrm{pH}$} & \multicolumn{2}{|l|}{ NS1 } & \multicolumn{2}{|l|}{ NS2 } & \multicolumn{2}{|l|}{ NS3 } \\
\hline & 4.4 & 6.7 & 4.4 & 6.7 & 4.4 & 6.7 \\
\hline \multicolumn{7}{|l|}{ Guest } \\
\hline Que & 95 & 95 & 80 & 92 & 85 & 94 \\
\hline Cur & $>97$ & $>97$ & $>97$ & 95 & $>97$ & $>97$ \\
\hline Phec & 92 & 93 & 93 & 95 & 90 & 92 \\
\hline
\end{tabular}

The latter observation is quite intriguing. In fact, based on the $\mathrm{p} K_{\mathrm{a}}$ values reported, it can be confidently assumed that the guest is in its undissociated form at both $\mathrm{pH}$ values. Conversely, materials NS2 and NS3 assume a positive charge density at the lowest $\mathrm{pH}$ value due to the partial protonation of the 1,2,3-triazole linker moieties. Inclusion efficiency is known to be significantly affected by Coulomb interactions and in general by a possible increase in the hydrophilic character of the NS due to the occurrence of a non-null charge density (caused by the protonation or deprotonation of ionisable groups as a function of the $\mathrm{pH}$ ). Therefore, this effect appears much more effective in the case of Que, which has a more compact and rigid molecule, compared to those in the cases of the more flexible Cur and Phec.

Preliminary absorption tests helped us to plan the preparation of the composites. Indeed, we reasoned that an excessive absorption efficiency, although suggesting possible high loading, could have also resulted in unsatisfactory release over time. Consequently, the choice relevant to the inclusion in a particular NS was made on the basis of a reasonable compromise between the two requirements. So, considering the data reported in Table 1, we decided to prepare three different composites by including Que into material NS1, Cur into NS2 and Phec into NS3. Loadings of the antioxidant agents into the nanosponges were carried out according to the literature (see Experimental) by equilibrating the NS materials with the guest species (a $4: 1 \mathrm{w} / \mathrm{w}$ starting ratio) in aqueous buffer at $\mathrm{pH}$ 4.4. The percent amount of guest actually present in the obtained composites was determined via UV-vis spectrophotometry after having eluted it with methanol. Loadings (w/w) were found to be as large as 7.3\% for the Que-NS1 composite, $17.3 \%$ for the Cur-NS2 composite and $12.9 \%$ for the Phec-NS3 composite. The composites were characterized using FT-IR spectroscopy. A careful inspection of the fingerprint region (Fig. 4) may in principle reveal the presence of the guest embedded into the NS matrix. Of course, the possible detection of signals attributable to the guest is heavily affected by the actual loading. In the case of the Que-NS1 composite, for instance, the presence of the guest is hardly identifiable (due to its low loading) by the very weak bands at ca. 1604 and $1324 \mathrm{~cm}^{-1}$. On the other hand, the presence of Cur in its composite is well outlined by at least three signals of the guest at 1629, 1605 and $1509 \mathrm{~cm}^{-1}$. Similarly, the Phec composite shows fairly intense bands at 1683, 1603, 1301, 1281, 1184, 1106 and $978 \mathrm{~cm}^{-1}$. It is worth noting that all these signals do not
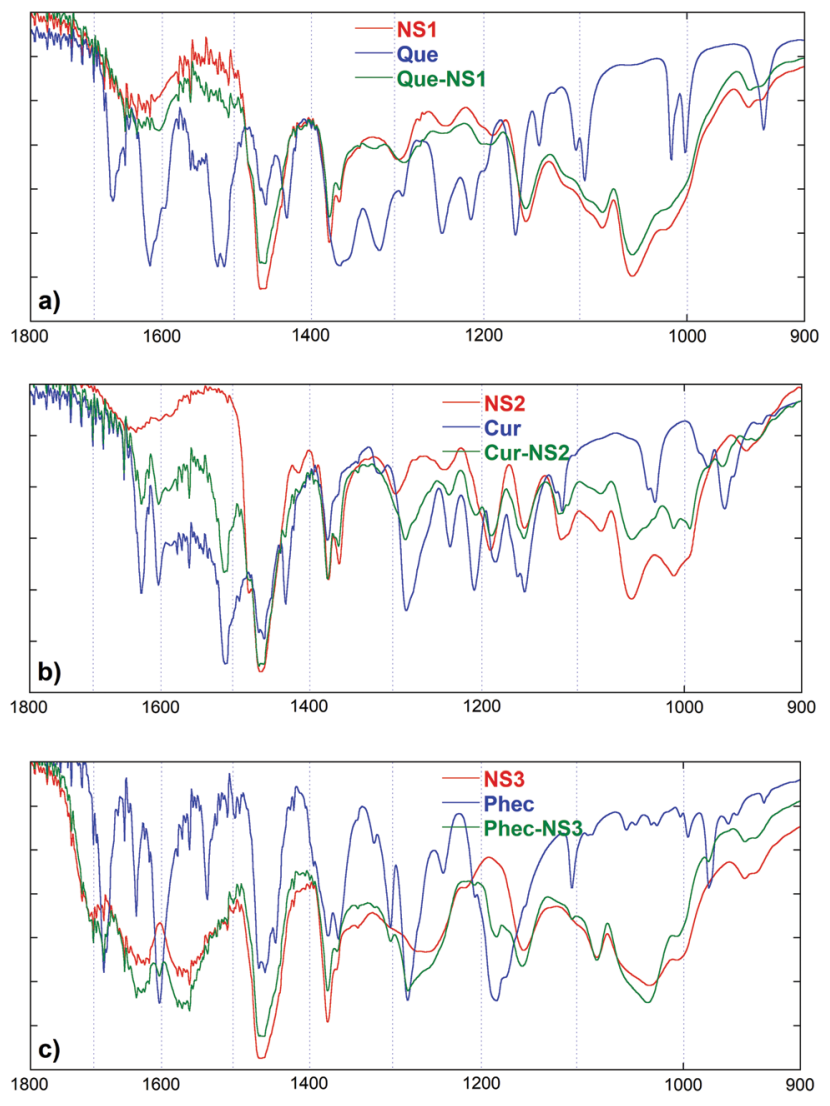

Fig. 4 FTIR spectra of the composites: Que-NS1 (a), Cur-NS2 (b) and Phec-NS3 (c).

show any significant shift with respect to the free guest, indicating the occurrence of only a weak non-specific interaction with the nanosponge matrix.

We then verified the release of the guest molecules by the composites under two different conditions (see Experimental): (i) aqueous solution buffered at $\mathrm{pH} 7.2$ and $37^{\circ} \mathrm{C}$, i.e. under conditions consistent with physiological systems; (ii) $\mathrm{pH} 7.4$ aqueous solution containing $10 \% \mathrm{v} / \mathrm{v}$ THF, which are standardized conditions used to study the antioxidant activity (see below). In the first case, we noticed that neither Que nor Cur is appreciably released even after $24 \mathrm{~h}$. Differently, Phec is actually released from its composite, though quite slowly (ca. 3.4\% within $6 \mathrm{~h}$ ). The relevant kinetic profile (Fig. 5, blue line) is the superimposition of a first order process with an apparent kinetic constant $k_{1}=(6 \pm 1) \times 10^{-4} \mathrm{~s}^{-1}$ and a zeroth order process with an apparent kinetic constant $k_{0}=(2.5 \pm 0.3) \times$ $10^{-10} \mathrm{M} \mathrm{s}^{-1}$. Noticeably, the occurrence of this peculiar profile can be easily explained admitting that first a little amount of the guest is quickly released from the outer layer of the nanosponge grains, and then a further amount is released from the inner region by a quite slow mass-transfer process. A similar behaviour was observed also under the second set of conditions. In more detail, only a little release amount is found for curcumin and quercetin (less than $2 \%$ ), and hence we were not able to perform a proper release kinetic experiment. By contrast (Fig. 5, red line), Phec is largely released (up to $c a$. 80\%) in a relatively short 


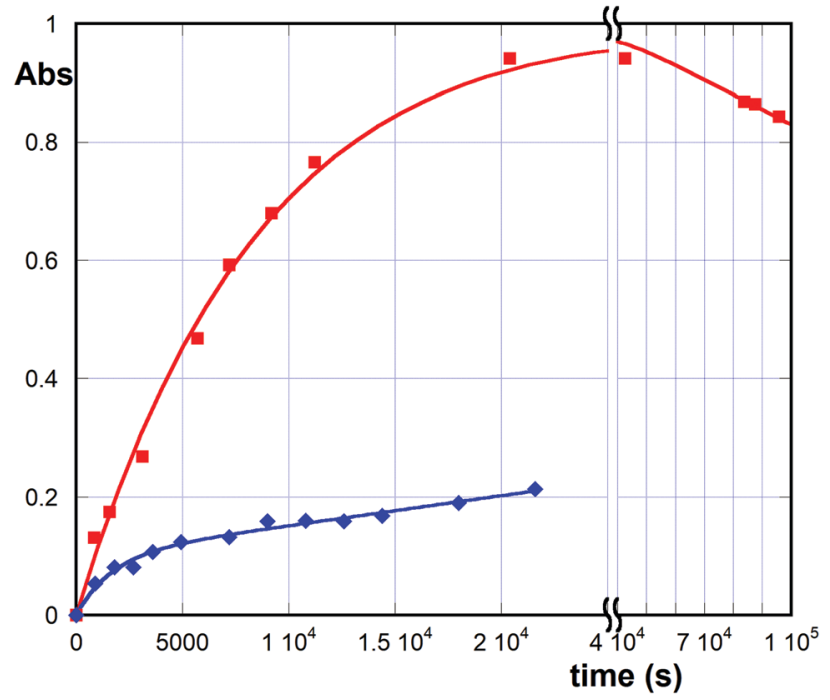

Fig. 5 Release kinetic profiles $\left(37^{\circ} \mathrm{C}\right)$ for the Phec-NS3 composite: (i) aqueous buffer at $\mathrm{pH} 7.2$ (blue) and (ii) THF/water $10 \% \mathrm{v} / \mathrm{v}$ buffered at $\mathrm{pH} 7.4$ (red). For Phec, $\lambda=328 \mathrm{~nm}$ and $\varepsilon=15800 \pm 800 \mathrm{M}^{-1} \mathrm{~cm}^{-1}$ in both media.

time $(4 \mathrm{~h})$ according to an apparent pseudo-first-order profile, with $k_{1}=(1.15 \pm 0.07) \times 10^{-4} \mathrm{~s}^{-1}$, which can be easily explained considering that the co-solvent THF molecules that are present compete with the guest for the binding sites of the NS material, so that mass-transfer is largely speeded up. However, Phec undergoes slow decomposition after release, as confirmed using a blank experiment on a solution of the free guest under the same conditions (decomposition rates were consistent for the two different experiments).

\section{Antioxidant activity assays}

The antioxidant activity was evaluated by measuring the inhibition rate constant $\left(k_{\mathrm{inh}}\right)$ and the stoichiometry $(n)$ of the reaction between the antioxidants and the alkylperoxyl radicals (ROO'), which are the most relevant radicals responsible for peroxidation in bioorganic systems. ${ }^{33}$ The values of $k_{\text {inh }}$ were determined by studying the inhibition of the thermally initiated autoxidation of tetrahydrofuran (THF), chosen as the reference oxidizable substrate (Scheme 1), under controlled conditions

$$
\begin{aligned}
& \text { Initiator } \stackrel{R_{\mathrm{i}}}{\longrightarrow} \mathrm{R}^{\cdot} \\
& \mathrm{R}^{\cdot}+\mathrm{O}_{2} \stackrel{k \approx 10^{9} \mathrm{M}^{-1} \mathrm{~s}^{-1}}{\longrightarrow} \mathrm{ROO} \cdot \\
& \mathrm{ROO}+\mathrm{RH} \stackrel{k_{\mathrm{p}}}{\longrightarrow} \mathrm{ROOH}+\mathrm{A}^{\circ} \\
& 2 \mathrm{ROO} \stackrel{k_{\mathrm{t}}}{\longrightarrow} \text { non-radical products } \\
& \mathrm{ROO}+\mathrm{AH} \stackrel{k_{\text {inh }}}{\longrightarrow} \mathrm{ROOH}+\mathrm{A}^{\circ} \\
& \mathrm{ROO}+\mathrm{A}^{\cdot} \stackrel{k \approx 10^{8} \mathrm{M}^{-1} \mathrm{~s}^{-1}}{\longrightarrow} \mathrm{ROOA}
\end{aligned}
$$

Scheme 1 General mechanism of the propagation/inhibition of the autoxidation reaction. $\mathrm{RH}$ represents the oxidizable substrate THF.
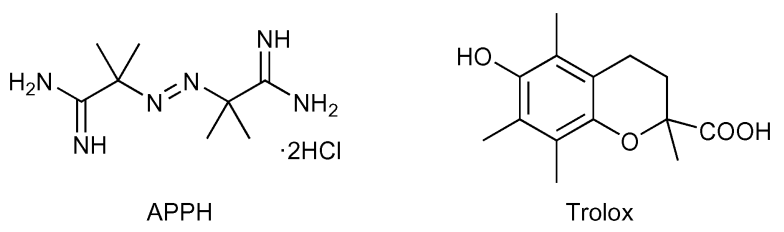

Fig. 6 Structures of AAPH and Trolox.

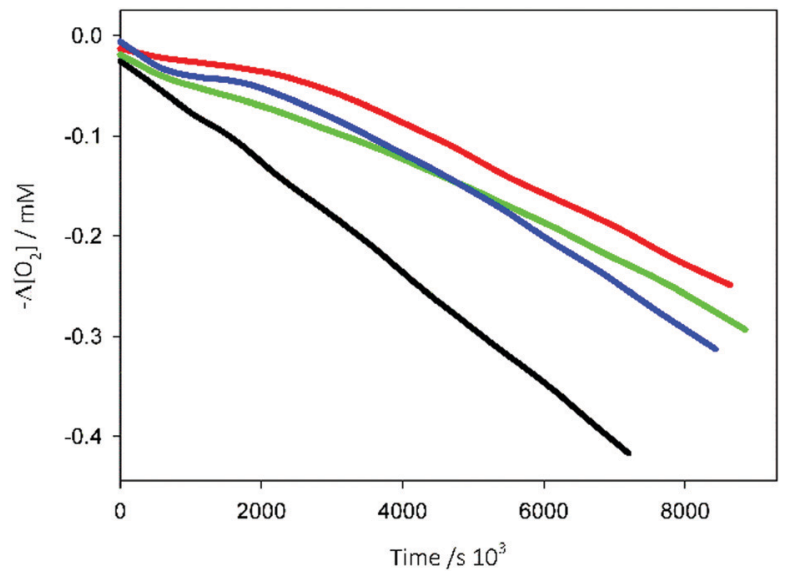

Fig. 7 Oxygen consumption during the autoxidation of THF (1.24 M) initiated by AAPH ( $25 \mathrm{mM})$ in $0.1 \mathrm{M}$ phosphate buffer at $\mathrm{pH} 7.4$ and $30{ }^{\circ} \mathrm{C}$ without inhibitors (black) or in the presence of samples $\left(4 \times 10^{-6} \mathrm{M}\right)$ : Phec (blue), Cur (green), and Que (red).

using $\mathrm{pH} 7.4$ phosphate buffer as the solvent. The reactions were performed at $30{ }^{\circ} \mathrm{C}$ using $2,2^{\prime}$-azobis-2-methylpropionamidine dihydrochloride (AAPH, Fig. 6) as the initiator, and the oxygen consumption $\left(\Delta\left[\mathrm{O}_{2}\right]\right)$ was measured using a differential pressure transducer. ${ }^{44-47}$

In the absence of antioxidants, THF autoxidation causes a linear $\mathrm{O}_{2}$ consumption (see for instance the black line in Fig. 7). In the presence of a good antioxidant, THF autoxidation displays an inhibition period, the duration of which depends on the antioxidant concentration and on the rate of radical generation by AAPH $\left(R_{\mathrm{i}}\right)$ as shown in eqn (1), while its slope is inversely proportional to the antioxidant efficacy. From the slope of $\mathrm{O}_{2}$ consumption during the inhibition period, we could obtain $k_{\text {inh }}$ values by using eqn (1), where AH represents the antioxidant, $R_{0}$ and $R_{\mathrm{inh}}$ are the slopes of $\mathrm{O}_{2}$ consumption without and with the antioxidant, respectively, $2 k_{\mathrm{t}}$ is the termination rate constant of THF $\left(6.6 \times 10^{7} \mathrm{M}^{-1} \mathrm{~s}^{-1}\right)^{46}$ and $R_{\mathrm{i}}$ is the rate of radical generation by AAPH. ${ }^{37}$ The stoichiometric coefficient $n$ represents the number of radicals trapped by each antioxidant molecule and was determined from the duration of the inhibition period $(\tau)$ and the antioxidant concentration using eqn (2). ${ }^{47}$

$$
\begin{gathered}
\frac{R_{0}}{R_{\text {inh }}}-\frac{R_{\text {inh }}}{R_{0}}=\frac{n k_{\text {inh }}[\mathrm{AH}]}{\sqrt{2 k_{\mathrm{t}} R_{\mathrm{i}}}} \\
\tau=\frac{n[\mathrm{AH}]}{R_{\mathrm{i}}}
\end{gathered}
$$


The antioxidant activities of Que, Cur and Phec alone were analysed, to assess their behaviour in the absence of the NSs. Because phenol stability is a critical issue at physiological $\mathrm{pH},{ }^{48}$ antioxidants were used at a relatively low concentration to avoid long reaction times. All three phenols caused an inhibition of THF oxidation (Fig. 7), accounting for the reaction with ROO• radicals. The $k_{\text {inh }}$ values obtained using this procedure are reported in Table 2. ${ }^{48}$ Que showed the highest $k_{\text {inh }}$, followed by Phec and then by Cur. All the three antioxidants, however, were less reactive than the antioxidant Trolox (Fig. 6), considered as the standard reference. It is worth pointing out that the $k_{\text {inh }}$ values measured herein are higher than those previously measured at a larger THF concentration $(3.1 \mathrm{M})$ for Trolox and Que $\left(k_{\mathrm{inh}}=4.1 \times 10^{5}\right.$ and $1.6 \times$ $10^{5} \mathrm{M}^{-1} \mathrm{~s}^{-1}$, respectively), ${ }^{46,49}$ indicating the importance of controlling the levels of organic co-solvents when measuring antioxidant activities in an aqueous environment.

Regarding the stoichiometry of radical trapping, it can be noticed that the $n$ coefficient reported in Table 2 in the case of Phec is significantly larger than that measured in non-aqueous systems (in chlorobenzene, $n \approx 2$ ). ${ }^{50}$ This could be explained by considering that after the reaction with the first two radicals, electrophilic quinonoid structures are formed, which are known to add solvent molecules generating new phenols having antioxidant activity (a possible mechanism is depicted in Scheme 2). ${ }^{51}$

We then investigated the antioxidant activity in the composites (Fig. 8). After having verified that the bare NSs have no appreciable activity of their own (dotted lines in Fig. 8), the observed plots of $\mathrm{O}_{2}$ consumption showed a marked inhibition of THF autoxidation due to the release of the phenols loaded into the NSs. The longer inhibition periods with respect to the experiments presented in Fig. 7 are due to the higher

Table 2 Antioxidant activity $\left(k_{\text {inh }}\right)$ and number of radicals trapped by each antioxidant molecule $(n)$ measured in inhibited autoxidation experiments at $30^{\circ} \mathrm{C}^{a}$

\begin{tabular}{lll}
\hline & $k_{\text {inh }}\left(\mathrm{M}^{-1} \mathrm{~s}^{-1}\right)$ & $n$ \\
\hline Trolox & $(2.5 \pm 0.6) \times 10^{6}$ & 2.0 \\
Que & $(7.6 \pm 0.5) \times 10^{5} b$ & $3.6 \pm 0.3^{b}$ \\
Cur & $(1.8 \pm 0.3) \times 10^{5}$ & $5.0 \pm 0.5$ \\
Phec & $(5.5 \pm 0.3) \times 10^{5}$ & $3.9 \pm 0.1$
\end{tabular}

${ }^{a}$ Average from triplicate experiments. ${ }^{b}$ From ref. 48.
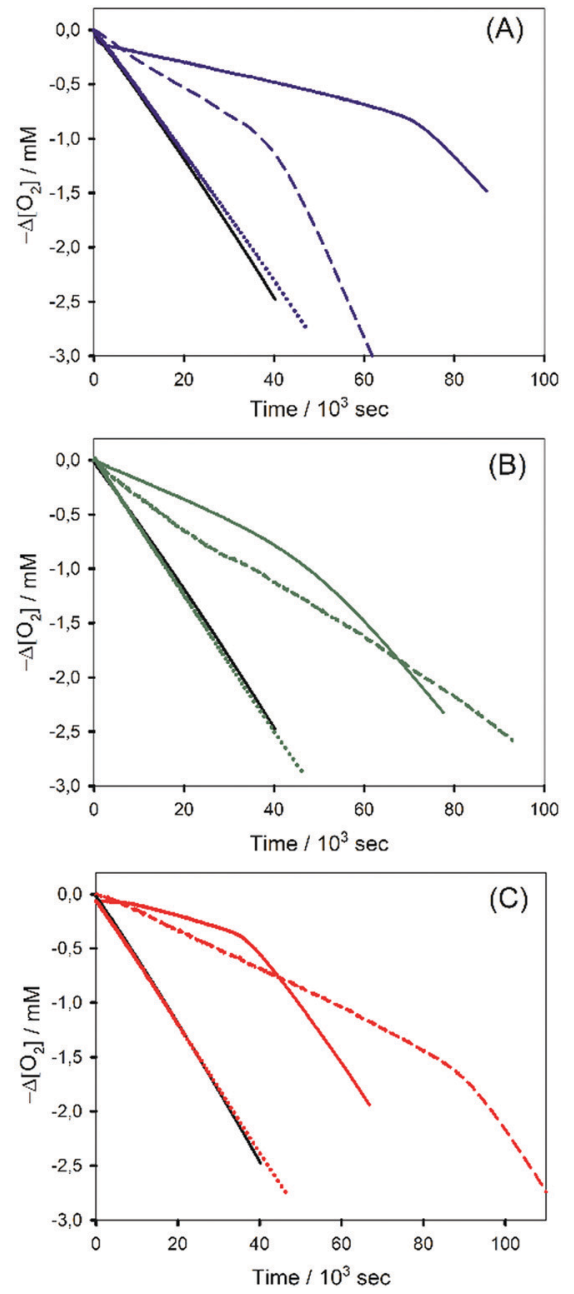

Fig. 8 Oxygen consumption for the autoxidation of THF (1.24 M) initiated by AAPH (25 mM) in $0.1 \mathrm{M}$ phosphate buffer at $\mathrm{pH} 7.4$ and $30{ }^{\circ} \mathrm{C}$ without inhibitors (black) or in the presence of (A) NS3 (blue dotted), Phec (blue dashed) and Phec-NS3 (blue solid); (B) NS2 (green dotted), curcumin (green dashed) and Cur-NS2 (green solid); and (C) NS1 (red dotted), quercetin (red dashed) and Que-NS1 (red solid). All antioxidant samples contained $9 \times 10^{-5} \mathrm{M}$ of phenols, and empty NSs are $0.25-0.38 \mathrm{mg} \mathrm{mL}^{-1}$.

concentrations of the polyphenols. Regression analysis using eqn (1) and (2) (data are collected in Table 3), where [AH] is the molar concentration of the sample of the polyphenols contained in the nanosponges, provided the $k_{\text {inh }}$ and $n$ values

a)

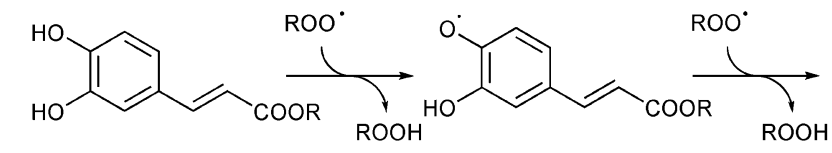<smiles>[R]OC(=O)/C=C/c1ccc(O)c(O)c1O</smiles>

b)<smiles>[R]OC(=O)/C=C/c1ccc([O-])c(O)c1</smiles><smiles>O=C(O)/C=C/c1ccc(Cl)c(O)c1</smiles>

Scheme 2 (a) Putative mechanism accounting for the trapping of more than two peroxyl radicals by Phec (adapted from ref. 51); (b) degradation of phenols in water solutions. 
Table 3 Antioxidant activity $\left(k_{\text {inh }}\right)$ and the number of radicals trapped by each antioxidant molecule $(n)$ measured in inhibited autoxidation experiments at $30{ }^{\circ} \mathrm{C}$ for phenols loaded on the nanosponges directly added in solution, at the same concentration ${ }^{a}$

\begin{tabular}{lcl}
\hline & $k_{\text {inh }}\left(\mathrm{M}^{-1} \mathrm{~s}^{-1}\right)$ & $n$ \\
\hline Que-NS1 & $(2.6 \pm 0.3) \times 10^{4}$ & $2.7 \pm 0.2$ \\
Cur-NS2 & $(9.0 \pm 0.3) \times 10^{3}$ & $4.0 \pm 0.1$ \\
Phec-NS3 & $(3.2 \pm 0.2) \times 10^{4}$ & $4.1 \pm 0.1$ \\
Conc. Que & $(1.1 \pm 0.2) \times 10^{4}$ & $6.2 \pm 0.2$ \\
Conc. Cur & $(3.0 \pm 0.4) \times 10^{3}$ & $>5$ \\
Conc. Phec & $(8.0 \pm 0.2) \times 10^{3}$ & $2.2 \pm 0.1$ \\
${ }^{a}$ Average from triplicate experiments. &
\end{tabular}

reported in Table 3. Noticeably, and as previously observed in the case of vitamin $\mathrm{C}$ loaded on halloysite nanotubes, ${ }^{52}$ the $k_{\text {inh }}$ values are smaller than those of free antioxidants because the antioxidant is released gradually from the sponges, where it is inactive.

In order to understand the application potential of the nanosponges as carriers of antioxidants, we investigated also the antioxidant activity of Phec, Que and Cur at high concentrations, identical to those used in the experiments where antioxidants are included in the nanosponges. The results reported in Table 3 show that the three phenols suffer a dramatic reduction of $k_{\text {inh }}$ upon increasing their concentration, which we explain as being due to their degradation that is known to occur in aqueous solution. ${ }^{52-54}$ It is worth mentioning here that the $k_{\text {inh }}$ values determined from the experiments reported in Fig. 8 are to be considered "apparent" inhibition constants, as the slopes of $\mathrm{O}_{2}$ consumption reported in Fig. 8 are influenced by the release from the nanosponges or by the reaction occurring during phenol degradation. Polyphenol decomposition occurs by the deprotonation of phenols and by the reaction of the phenolate with $\mathrm{O}_{2}$, to afford phenoxyl radicals and superoxide, which propagates the oxidative chain (Scheme 2b). ${ }^{52}$ The results (illustrated in Fig. 8) show that phenols included into nanosponges provided in all cases a more efficient trapping of $\mathrm{ROO}^{\bullet}$ (i.e. a smaller slope of $\mathrm{O}_{2}$ consumption and, in the case of Que, longer inhibition) than phenols at the same concentration, free in solution. Interestingly, the results reported in Fig. 8 show that polyphenol degradation does not necessarily correspond to a reduction of the inhibition period. Indeed, products possessing phenolic moieties having a residual antioxidant may be formed, as in the case of curcumin in which a phenolic heterocycle is formed after degradation. ${ }^{55}$ Therefore, we can say that nanosponges not only allow a gradual release of the previously loaded phenols, but also protect them from external agents, increasing their effectiveness over time.

\section{Experimental}

\section{Materials and instrumentation}

All the reagents and materials needed were used as purchased (Sigma-Aldrich) without further purification. The FT-IR spectra of the nanosponge composites were acquired using an AGILENT Technologies Cary 630 FTIR spectrometer. UV-vis spectra were recorded using a Beckmann-Coulter DU800 spectrophotometer.
The nanosponges $\mathrm{NS} 1,{ }^{42} \mathrm{NS}^{22}$ and $\mathrm{NS}^{23}$ were prepared, purified and characterized according to literature reports. For information completeness, NS1 contained $0.61 \mathrm{mmoles}^{-1}$ of $\beta C D$ units, NS2 contained 0.46 mmoles $\mathrm{g}^{-1}$ of $\mathrm{A}_{7} \mathrm{CD}$ units and 0.50 mmoles $\mathrm{g}^{-1}$ of $\mathrm{P}_{4} \mathrm{C}[4] \mathrm{A}$ units, and NS3 contained 0.25 mmoles $\mathrm{g}^{-1}$ of $\mathrm{A}_{7} \mathrm{CD}$ units and 0.85 mmoles $\mathrm{g}^{-1}$ of $\mathrm{P}_{4} \mathrm{C}[4] \mathrm{A}$ units.

The composites were prepared as follows. The plain nanosponge $(80.0 \mathrm{mg}$ ) was placed in a vial, wetted with a few drops of methanol, and suspended in $20 \mathrm{~mL}$ of an aqueous acetic buffer (0.05 M, pH 4.4). Then $20.0 \mathrm{mg}$ of the guest was dissolved in $1 \mathrm{~mL}$ of methanol and the solution was added slowly to the NS suspension under gentle magnetic stirring. The system was kept under stirring at r.t. overnight, and then the product was vacuum filtered off, rapidly washed with drops of ice-cold methanol, and finally dried in vacuo at $50{ }^{\circ} \mathrm{C}$ over phosphorus pentoxide for $2 \mathrm{~h}$.

\section{Sequestration and release tests}

Sequestration tests were performed as follows. A carefully weighed amount $(4.00 \pm 0.05 \mathrm{mg})$ of the nanosponge material was placed in a vial and mixed with $2 \mathrm{~mL}$ of a $50 \mathrm{mM}$ solution of the guest in aqueous buffer $(I=0.05 \mathrm{M})$ at the proper $\mathrm{pH}$ value (acetate buffer for $\mathrm{pH} 4.4$, phosphate buffer for $\mathrm{pH}$ 6.7). The sample was briefly sonicated to facilitate the dispersion of the material and then mechanically shaken for $90 \mathrm{~min}$. The sample was then centrifuged (5500 rpm for $15 \mathrm{~min}$ ); the supernatant liquor was carefully pipetted and analyzed (UV-vis) to determine the residual concentration in solution, from which the percent absorption was calculated by trivial algebraic passages.

The release tests were performed as follows. A carefully weighed amount $(10.00 \pm 0.05 \mathrm{mg})$ of the composite was placed in a dialysis membrane (Medicell International Ltd; MWCO 1214000 with a diameter of $21.5 \mathrm{~mm}$ ) and dispersed in $1 \mathrm{~mL}$ of aqueous phosphate buffer solution (0.05 $\mathrm{M}$ and $\mathrm{pH} 7.2$; at $\mathrm{pH}$ 7.4 in buffer solution with $10 \%$ THF). The membrane was then placed in a round bottom flask containing $20 \mathrm{~mL}$ of the release medium at $37{ }^{\circ} \mathrm{C}$ under magnetic stirring. At fixed times, $1 \mathrm{~mL}$ of the release medium was withdrawn and analyzed spectrophotometrically to determine the concentration of the guest released. Notably, in order to keep the volume of the sample constant, $1 \mathrm{~mL}$ of fresh buffer was added each time to replace the withdrawn one.

\section{Antioxidant activity}

The antioxidant activity was evaluated by measuring the $\mathrm{O}_{2}$ consumption during the autoxidation of tetrahydrofuran (THF) initiated by the hydrosoluble azo-initiator AAPH (25 mM) at $30{ }^{\circ} \mathrm{C}$. The initiation rate, $R_{\mathrm{i}}$, was determined by the inhibition method using ( \pm )-6-hydroxy-2,5,7,8-tetramethylchromane2-carboxylic acid (Trolox) as the reference antioxidant: $R_{\mathrm{i}}=$ $2[\mathrm{PMHC}] / \tau$, where $\tau$ is the length of the induction period $\left(R_{\mathrm{i}}=6.2 \times 10^{-9} \mathrm{M} \mathrm{s}^{-1}\right){ }^{56}$ The concentration of $\mathrm{O}_{2}$ was measured using an optical oxygen meter (FireStingO2, PyroScience $\mathrm{GmbH})^{57}$ calibrated by performing a reference THF autoxidation. 


\section{Conclusions}

The goal of this work is represented by the possibility of performing a controlled release in an aqueous medium of natural antioxidants that are sparingly water soluble. In more detail, cyclodextrin- and calixarene-based nanosponges were prepared for the delivery of natural antioxidants, quercetin, curcumin and phenethyl caffeate. The obtained composites were evaluated for guest solubility, guest release and antioxidant properties.

Our results positively demonstrate that the use of our nanosponge materials is an interesting approach for the protection and controlled disposal of natural polyphenolic antioxidants. The structure of nanosponges can be easily modulated by changing the relative proportions of the host molecules and of the linkers, to obtain optimal loading and release properties. Kinetic experiments on the reaction with $\mathrm{ROO}^{-}$radicals indicate that the inclusion into nanosponges increases the antioxidant activity by blocking the pro-oxidant effect due to phenol degradation. The flexibility of the synthetic method allows us to envisage many future developments involving both the nanosponge scaffold (biocompatibility, biodegradation, material size) and the radical trapping moieties (such as integrating covalently and noncovalently loaded antioxidants with different mechanisms of action). In conclusion, we believe that opportunely designed nanosponges have the potential to give a significant contribution to the development of new highly active antioxidants.

\section{Conflicts of interest}

There are no conflicts to declare.

\section{Acknowledgements}

The funding from Bologna University is gratefully acknowledged.

\section{Notes and references}

1 V. Conti, V. Izzo, G. Corbi, G. Russomanno, V. Manzo, F. De Lise, A. Di Donato and A. Filippelli, Front. Pharmacol., 2016, 7, 24, DOI: 10.3389/fphar.2016.00024.

2 G. Bjørklund and S. Chirumbolo, Nutrition, 2017, 33, 311-321.

3 J.-Z. Wang, R.-Y. Zhang and J. Bai, Int. J. Cardiol., 2020, 312, 137-138.

4 C. G. Fraga, K. D. Croft, D. O. Kennedy and F. A. TomásBarberán, Food Funct., 2019, 10, 514-528.

5 A. Yashin, Y. Yashin, X. Xia and B. Nemzer, Antioxidants, 2017, 6, 70.

6 F. Aqil, R. Munagala, J. Jeyabalan and M. V. Vadhanam, Cancer Lett., 2013, 334, 133-141.

7 P. Khadka, J. Ro, H. Kim, I. Kim, J. T. Kim, H. Kim, J. M. Cho, G. Yun and J. Lee, Asian J. Pharm. Sci., 2014, 9, 304-316.

8 R. Conte, V. Marturano, G. Peluso, A. Calarco and P. Cerruti, Int. J. Mol. Sci., 2017, 18, 709, DOI: 10.3390/ijms18040709.
9 A. Fontana, S. Guernelli, A. Di Crescenzo, P. Di Profio, F. Palomba, L. De Crescentini, A. Baschieri and R. Amorati, J. Mol. Liq., 2019, 293, 111465.

10 A. Vaiserman, A. Koliada, A. Zayachkivska and O. Lushchak, Front. Bioeng. Biotechnol., 2020, 7, 1-19.

11 M. Massaro, C. G. Colletti, B. Fiore, V. La Parola, G. Lazzara, S. Guernelli, N. Zaccheroni and S. Riela, Gold nanoparticles stabilized by modified halloysite nanotubes for catalytic applications, Appl. Organomet. Chem., 2019, 33, 1-11.

12 P. Ganesan, G. Karthivashan, S. Y. Park, J. Kim and D. K. Choi, Int. J. Nanomed., 2018, 13, 6109-6121.

13 P. Lo Meo, F. Mundo, S. Terranova, P. Conte and D. Chillura Martino, J. Phys. Chem. B, 2020, 124, 1847-1857.

14 R. Cavalli, F. Trotta and W. Tumiatti, J. Inclusion Phenom. Macrocyclic Chem., 2006, 56, 209-213.

15 F. Trotta, Cyclodextrins in Pharmaceutics, Cosmetics, and Biomedicine, John Wiley \& Sons, Inc., 2011, pp. 323-342, DOI: $10.1002 / 9780470926819 . c h 17$.

16 S. Selvamuthukumar, S. Anandam, K. Kannan and R. Manavalan, J. Pharm. Pharm. Sci., 2012, 15, 103-111.

17 P. K. Shende, F. Trotta, R. S. Gaud, K. Deshmukh, R. Cavalli and M. Biasizzo, J. Inclusion Phenom. Macrocyclic Chem., 2012, 74, 447-454.

18 A. L. Taka, K. Pillay and X. Y. Mbianda, Carbohydr. Polym., 2017, 159, 94-107.

19 M. Russo, M. L. Saladino, D. Chillura Martino, P. Lo Meo and R. Noto, RSC Adv., 2016, 6, 49941-49953.

20 A. Spinella, M. Russo, A. Di Vincenzo, D. C. Martino and P. Lo Meo, Beilstein J. Org. Chem., 2018, 14, 1498-1507.

21 V. Cinà, M. Russo, G. Lazzara, D. Chillura Martino and P. Lo Meo, Carbohydr. Polym., 2017, 157, 1393-1403.

22 P. Lo Meo, G. Lazzara, L. Liotta, S. Riela and R. Noto, Polym. Chem., 2014, 5, 4499-4510.

23 M. Massaro, V. Cina, M. Labbozzetta, G. Lazzara, P. Lo Meo, P. Poma, S. Riela and R. Noto, RSC Adv., 2016, 6, 50858-50866.

24 A. Di Vincenzo, M. Russo, S. Cataldo, D. Milea, A. Pettignano and P. Lo Meo, ChemistrySelect, 2019, 4, 6155-6161.

25 R. M. Fontana, N. Milano, L. Barbara, A. Di Vincenzo, G. Gallo and P. L. Meo, ChemistrySelect, 2019, 4, 9743-9747.

26 K. A. Ansari, P. R. Vavia, F. Trotta and R. Cavalli, AAPS PharmSciTech, 2011, 12, 279-286.

27 N. K. Dhakar, A. Matencio, F. Caldera, M. Argenziano, R. Cavalli, C. Dianzani, M. Zanetti, J. M. López-Nicolás and F. Trotta, Pharmaceutics, 2019, 11, 545.

28 A. Matencio, N. K. Dhakar, F. Bessone, G. Musso, R. Cavalli, C. Dianzani, F. García-Carmona, J. M. López-Nicolás and F. Trotta, Carbohydr. Polym., 2020, 231, 115763.

29 R. Pushpalatha, S. Selvamuthukumar and D. Kilimozhi, J. Drug Delivery Sci. Technol., 2017, 39, 362-371.

30 S. Anandam and S. Selvamuthukumar, J. Porous Mater., 2014, 21, 1015-1023.

31 Z. Rezaei, S. Khabnadideh, K. Pakshir, Z. Hossaini, F. Amiri and E. Assadpour, Eur. J. Med. Chem., 2009, 44, 3064-3067. 
32 M. Ramírez-Ambrosi, F. Caldera, F. Trotta, L. Á. Berrueta and B. Gallo, J. Inclusion Phenom. Macrocyclic Chem., 2014, 80, 85-92.

33 Z. A. M. Zielinski and D. A. Pratt, J. Org. Chem., 2017, 82, 2817-2825.

34 M. C. Foti, J. Agric. Food Chem., 2015, 63, 8765-8776.

35 R. Shah, L. A. Farmer, O. Zilka, A. T. M. Van Kessel and D. A. Pratt, Cell Chem. Biol., 2019, 26, 1594.

36 M. Massaro, S. Riela, S. Guernelli, F. Parisi, G. Lazzara, A. Baschieri, L. Valgimigli and R. Amorati, J. Mater. Chem. B, 2016, 4, 2229-2241.

37 M. Massaro, R. Amorati, G. Cavallaro, S. Guernelli, G. Lazzara, S. Milioto, R. Noto, P. Poma and S. Riela, Colloids Surf., B, 2016, 140, 505-513.

38 C. Viglianisi, A. Scarlini, L. Tofani, S. Menichetti, A. Baschieri and R. Amorati, Sci. Rep., 2019, 9, 17219.

39 A. Rauf, M. Imran, I. E. Orhan and S. Bawazeer, Trends Food Sci. Technol., 2018, 74, 33-45.

40 V. Pittalà, L. Salerno, G. Romeo, R. Acquaviva, C. Di Giacomo and V. Sorrenti, Curr. Med. Chem., 2018, 25, 4827-4836.

41 R. V. Patel, B. M. Mistry, S. K. Shinde, R. Syed, V. Singh and H.-S. Shin, Eur. J. Med. Chem., 2018, 155, 889-904.

42 L. D. Wilson, M. H. Mohamed and C. L. Berhaut, Materials, 2011, 4, 1528-1542.

43 L. D. Wilson, M. H. Mohamed and J. V. Headley, J. Colloid Interface Sci., 2011, 357, 215-222.

44 R. Amorati, A. Baschieri and L. Valgimigli, J. Chem., 2017, 6369358.
45 R. Amorati and L. Valgimigli, J. Agric. Food Chem., 2018, 66, 3324-3329.

46 R. Amorati, A. Baschieri, G. Morroni, R. Gambino and L. Valgimigli, Chem. - Eur. J., 2016, 22, 7924-7934.

47 E. T. Denisov and I. V. Khudyakov, Chem. Rev., 1987, 87, 1313-1357.

48 M. C. Foti, R. Amorati, A. Baschieri and C. Rocco, Biophys. Chem., 2018, 243, 17-23.

49 R. Amorati, A. Baschieri, A. Cowden and L. Valgimigli, Biomimetics, 2017, 2, 9.

50 C. Spatafora, C. Daquino, C. Tringali and R. Amorati., Org. Biomol. Chem., 2013, 11, 4291-4294.

51 S. Saito, H. Gao and J. Kawabata, Helv. Chim. Acta, 2006, 89, 821-831.

52 A. Baschieri, R. Amorati, T. Benelli, L. Mazzocchetti, E. D'Angelo and L. Valgimigli, Antioxidants, 2019, 8, 30, DOI: 10.3390 /antiox8020030.

53 A. Schneider, J. Hemmerlé, M. Allais, J. Didierjean, M. Michel, M. d'Ischia and V. Ball, ACS Appl. Mater. Interfaces, 2018, 10, 7574-7580.

54 Y. J. Moon, L. Wang, R. DiCenzo and M. E. Morris, Biopharm. Drug Dispos., 2008, 29, 205-217.

55 C. Schneider, O. N. Gordon, R. L. Edwards and P. B. Luis, J. Agric. Food Chem., 2015, 63, 7606-7614.

56 L. R. C. Barclay and K. U. Ingold, J. Am. Chem. Soc., 1981, 103, 6478-6485.

57 F. Mollica, M. Lucarini, C. Passerini, C. Curati, S. Pavoni, L. Bonoldi and R. Amorati, Antioxidants, 2020, 9, 399. 